\title{
Cellular Phone Irradiation of the Head Affects Heart Rate Variability Depending on Inspiration/Expiration Ratio
}

\author{
SZABOLCS BÉRES ${ }^{1}$, ÁDÁM NÉMETH ${ }^{1}$, ZÉNÓ AJTAY ${ }^{1}$, \\ ISTVÁN KISS ${ }^{2}$, BALÁZS NÉMETH ${ }^{2}$ and LÁSZLÓ HEJJEL ${ }^{1}$ \\ ${ }^{1}$ Heart Institute, University of Pécs, Pécs, Hungary; \\ ${ }^{2}$ Department of Public Health Medicine, Medical School, University of Pécs, Pécs, Hungary
}

\begin{abstract}
Background: Mobile phones may have harmful health effects and clinical examinations report ambiguous results of exposure concerning neurophysiological and cardiovascular actions. Materials and Methods: This study investigated heart rate asymmetry (HRA) and heart rate variability (HRV) parameters with 1:2 and 1:1 metronomepaced inspiration/expiration ratios during short-term $1,800 \mathrm{MHz}$ GSM cellular phone exposure in 20 healthy volunteers. Results: Significant HRA changes by Porta and Guzik indices were not found on exposure compared to sham exposure. Time-domain HRV parameters on exposure showed significant differences at 1:1 paced, but not at 1:2 paced breathing compared to sham exposure. A mild post-exposure effect was observed regarding root mean square of successive RR-differences. Conclusion: The findings reflect persisting acute effects of GSM handset emission on the autonomic nervous system. Exploring its influences on health status and survival needs further studies. Symmetrical breathing can be used as a sensitizing factor in other HRV/HRA analysis studies.
\end{abstract}

Evolution of species has taken place in the presence of natural ionizing- and non-ionizing electromagnetic radiation, including sunlight, cosmic radiation, natural radioactivity and atmospheric electromagnetic phenomena, among others (1). There is increasing concern regarding the potentially harmful biological effects of exposure to electromagnetic fields (EMF), especially on humans, from military, industrial and commercial wireless telecommunication systems. Mobile

This article is freely accessible online.

Correspondence to: Dr. László Hejjel, Heart Institute, University of Pécs, Pécs, Hungary, Ifjúság str. 13., Pécs, H-7624, Hungary. Tel: +36 72536001, e-mail: hejjel.laszlo@pte.hu

Key Words: Heart rate variability, heart rate asymmetry, cellular phone, microwave radiation. phones have become popular and indispensable in our everyday life. The first generation analogue network was replaced in 1991 by the digital Global System for Mobile Communication (GSM), which had more than $90 \%$ market share still in 2014 in spite of the introduction of the thirdgeneration Universal Mobile Telecommunication System (UMTS) in 2001 and the fourth-generation Long-term Evolution (LTE) in 2009, with increasingly improved transmission capacity (2). All three generations of mobile communication standards are simultaneously present in Hungary in 2017 (3).

The mobile communication system consists of a cellular network of base and mobile stations (cellular phone or handset). The latter emits significantly less microwave power, however, its long-term close proximity to the head and torso results in more significant exposure in the general (not professional) population. The possible biological effects of microwave radiation are related to the energy absorption by living cells and their interactions at the molecular, cellular, tissue and organism level. Since the relative permeability of biological materials is around unity, independently of the exposure frequency, whereas their relative permittivity is $10-200$, the magnetic rather than electric component of EMF may be responsible for any biological effects (4).

The direct biophysical mechanism of the interaction of EMF and living tissues is still not clear; however, there are several theories (1). Membranes play a crucial role in biology by ensuring compartmentalization and interaction of the inner and outer cellular spaces at the same time. The interplay between membrane potential and membrane conductivity regulates cell functions, and excitation of neurons and contractile cells among others. A direct membrane-polarizing effect of microwave radiation can be excluded since the average capacity of the cell membrane $(1 \mu \mathrm{F} / \mathrm{cm})$ is considered as a short circuit above $\sim 1 \mathrm{MHz}$, hence there is no voltage drop and energy dissipation induced across the membrane itself (5). However, a low-frequency modulated radiofrequency field can act differently. Moreover, 
considering the resting membrane potential of $-70 \mathrm{mV}$ results in an electric field gradient across the $5 \mathrm{~nm}$-thick cell membrane of $\mathrm{E} \approx 14 \times 10^{6} \mathrm{~V} / \mathrm{m}$, the external $\mathrm{EMF}$ used in communication are negligibly weak to be able to directly act on membrane potential (6). Furthermore, the quantum (ionizing) effect of microwave radiation at the molecular level can be disregarded considering the energy carried by photons with a frequency of $0.300-300 \mathrm{GHz}$ would be $1.24 \times 10^{-6}$ to $1.24 \times 10^{-3} \mathrm{eV}$. This amount of energy is well below the ionizing threshold of biological molecules $(\sim 13.6 \mathrm{eV})$ and even smaller than the $0.027 \mathrm{eV}$ energy of Brownian motion at $37^{\circ} \mathrm{C}$ (4). Today the most widely accepted and explored theory for interaction is based on the thermal effects of microwave radiation. The current safety limits are established based on this; however, there are several biological phenomena that cannot be explained simply by warming of tissues, and temperature changes cannot be detected at all behind some observed biological effects $(1,7)$. The physical basis for thermal effects is excitation by rotational resonance of dipole molecules induced by sinusoidal EMF, which is resisted by the molecular moment of inertia and friction with the surrounding particles, which increases the thermal energy of the object, resulting in its warming up. In the microwave range, water and similar small dipolar molecules are the target of resonant frequency, although conformational changes of proteins via their bound water or molecular arms can also be considered targets. Depending on the inhomogeneity of the tissues, local hot-spots can arise, and due to the temperature-dependent nature of relative permittivity, focal thermal breakdown can occur (4).

The present applicable standard of maximal transmission power of a hand unit is $1.0 \mathrm{~W}$ at GSM $1800 / 1900$ and $2.0 \mathrm{~W}$ at GSM 850/900 (8). The European Union specific absorption rate (SAR) limit for the public is $2.0 \mathrm{~W} / \mathrm{kg}$ averaged over $10 \mathrm{~g}$ of tissue, but in a few countries the limit is $1.6 \mathrm{~W} / \mathrm{kg}$ averaged over $1 \mathrm{~g}$ of tissue (9).

Exposure to EMF may have negative health effects even below the power defined in safety standards. Investigations into possible adverse biological effect of cellular phones are focused on the central nervous system (CNS) and inner ear, because most of the microwave radiation is absorbed here due to the anatomical proximity to the signal source (10-15). Conclusions of human trials on the impact of EMF on the autonomic nervous system are controversial (16-19).

HRV analysis is a non-invasive assessment of the actions of the autonomic neuroendocrine system on the sinus node via the beat-to-beat fluctuations of the heart rate. This method is considered valuable in the prediction of progression and outcome in several diseases (20-22). Atlas et al. found no difference in HRV in 35 healthy volunteers breathing spontaneously during 10-minute exposure to EMF from a commercial mobile phone forced at $2 \mathrm{~W}$ output peak power at $900 \mathrm{MHz}$ (23). Heart rate asymmetry (HRA) is a relatively novel marker of HRV, based on the Poincaré-plot. This asymmetry can be quantified as Guzik index (24) or Porta index (25), among others. HRA reflects the dynamics of respiratory arrhythmia, namely temporal asymmetry of pulse rate acceleration and deceleration as a consequence of inspiration/expiration period ratio, first published by our research group in 2012 (26): Change in inspiration/expiration ratio by double-paced breathing from $1: 2$ to $1: 1$ or $2: 1$ is followed by corresponding change of HRA parameters. HRA measures can be more sensitive markers of respiration-heart rate coupling than standard HRV parameters; additionally, paced breathing 'stress-situation' requiring auditory and cortical functions also acts as a sensitizing factor.

In the available literature, there is no publication on the effect of cellular phone exposure on HRA parameters and at different paced inspiration/expiration period ratios. The aim of present study was to examine the influence of the acute effects of pulsed microwave irradiation from a commercial cellular phone on HRV and HRA parameters during various double-paced breathing patterns in healthy volunteers in randomized double-blind repeated-measures crossover design.

\section{Materials and Methods}

The study was approved by the Regional Research Ethics Committee (approval number: 2013/4747) and was conducted in accordance with the Declaration of Helsinki and its later amendments. Twenty healthy volunteers (14 female and six male) were enrolled. Informed consent was obtained from all individual participants included in the study. The mean age was 25.2 (range 21 to 32) years. The majority of the volunteers did not smoke (17 altogether), two people smoked fewer than 10 cigarettes/day, and one person smoked more than 10 cigarettes/day. Sixteen volunteers consumed alcohol occasionally, while four did not consume alcohol at all. Regarding coffee consumption, the population was heterogeneous: six did not consume at all, seven occasionally and seven regularly drank coffee. The mean body mass index (BMI) of the group was within the normal range: $21.8 \pm 2.5 \mathrm{~kg} / \mathrm{m}^{2}$. The participants were not allowed to consume alcohol, coffee or cigarettes for 4 hours before the test.

A commercial Nokia 6230i mobile telephone (Nokia Corporation, Helsinki, Finland) was used for radiofrequency exposure working on an $1,800 \mathrm{MHz}$ GSM network $(217 \mathrm{~Hz}$ pulse rate; $0.577 \mu$ s pulse width, actual emissions during the experiment were not measured) with SAR value of $0.70 \mathrm{~W} / \mathrm{kg}$ at the head (27). The call to the mobile phone was initiated from a landline unit in a neighbouring room until automatic disconnection at about 1 minute and $25 \mathrm{sec}$, and then the number was redialled three more times over a 6-minute period. The participants had no information on the emission of the phone since it was in silent mode and was totally covered by an elastic bandage.

During each experimental stage, a 6-min-long single channel electrocardiogram (ECG) and respiration signal were recorded by a microcontroller-based, battery-powered, handheld data acquisition system (László Hejjel, Pécs, Hungary). An ultra-rapid thermistor probe was fixed under the volunteer's nose for monitoring respiration. The thermistor sensed the cooling and warming of the 

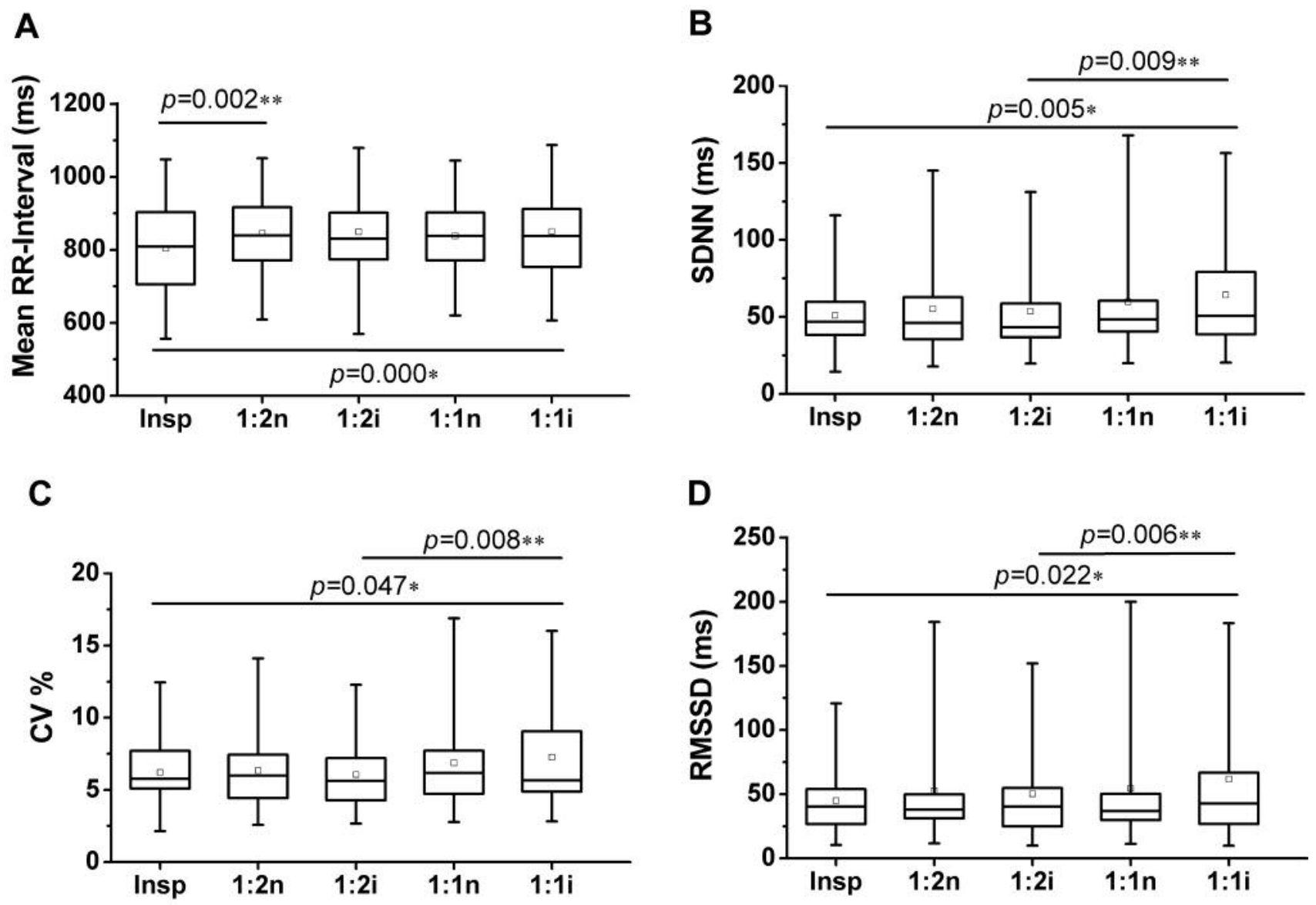

Figure 1. Box and whisker diagrams of the time domain parameters. A: Mean time between two R-waves on the ECG (RR interval). B: Standard deviation of RR intervals (SDNN). C: Coefficient of variation (CV\%). D: Root mean square of successive RR interval differences (RMSSD). Boxes: Lower and upper quartiles, horizontal line: median value, whiskers: minimum and maximum values, square: mean value. *Friedman test p-value, **post-hoc Wilcoxon's paired-sample test p-values, only significant differences are indicated. Insp: Only inspiration paced without exposure; 1:1 symmetrically paced inspiration/expiration; $1: 2$ asymmetrically paced inspiration/expiration; $i$ : with irradiation; $n:$ without irradiation.

flowing air during inhalation and exhalation, respectively. The instrument also contained a metronome to trigger inspiration and expiration with two different frequency beeps. Standard 300-second tachograms (22) were extracted from the ECG records by ECGRdet v2.4 (László Hejjel), while the HRV analysis was carried out with Varian v2.2 (László Hejjel). The mean time between two R-waves on the ECG (RR interval; MeanRR), standard deviation of normalto-normal RR-intervals (SDNN), coefficient of variation $(\mathrm{CV} \%=\mathrm{SDNN} / \mathrm{MeanRR})$, root mean square of successive RRinterval differences (RMSSD) in the time domain $(21,22)$; highfrequency (HF) integral in the range of $0.15-0.40 \mathrm{~Hz}$ (in normalized units), low-frequency (LF) component at $0.01-0.155 \mathrm{~Hz}$ (in normalized units) and LF/HF were analysed by fast Fourier transformation in the frequency domain $(21,22)$; Porta index and Guzik index $(24,25)$ were also computed as HRA parameters.

The study was carried out at our Institution in 2014 in the late afternoon, in quiet circumstances. The volunteers lay in a comfortable supine position with head elevated at $30^{\circ} 15$ minutes prior to data acquisition to allow orthostatic adaptation. The ECG electrodes along with the thermistor probe were placed on the subject, and the mobile phone was fixed on their right ear with an elastic bandage imitating its position during use. The subjects were educated regarding metronome-controlled respiration during this adaptation period. Talking and movements were not allowed, since these can influence HRV parameters (28).

Five consecutive 360-second-long stages were recorded for each volunteer. A $\mathrm{T}=4.5 \mathrm{~s}$ breathing cycle $(0.22 \mathrm{~Hz})$ was ensured by participants breathing according to the built-in beeper. Since previous studies have already demonstrated that single metronomecontrolled versus spontaneous breathing does not differ as regards of HRV parameters, we did not investigate spontaneous respiration here in order to reduce the study duration and hence the stress on the participants $(29,30)$. During the first stage, only inhalation was according to the metronome. In the second and fourth stages, inhalation and exhalation were triggered with beeps of two different frequencies, in an asymmetrical manner at a 1:2 (physiological) inspiration:expiration ratio. During the third and fifth stages, symmetrical breathing was paced, i.e. 1:1 inhalation:exhalation 


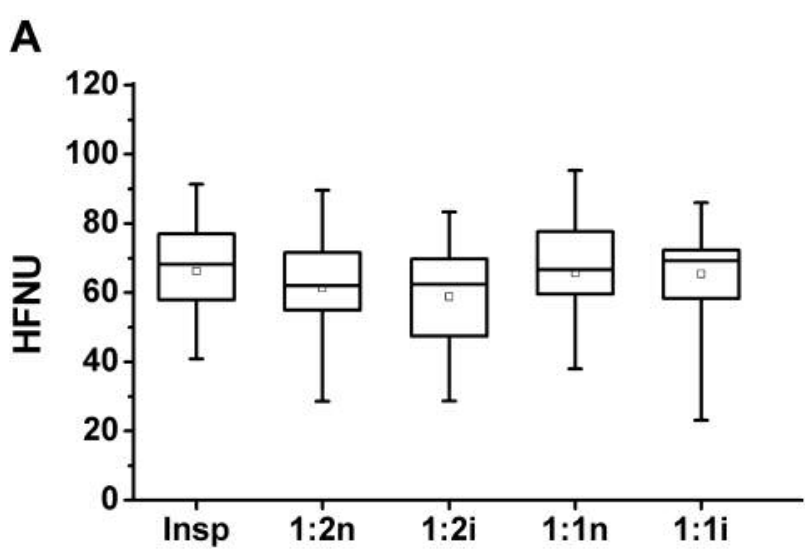

B

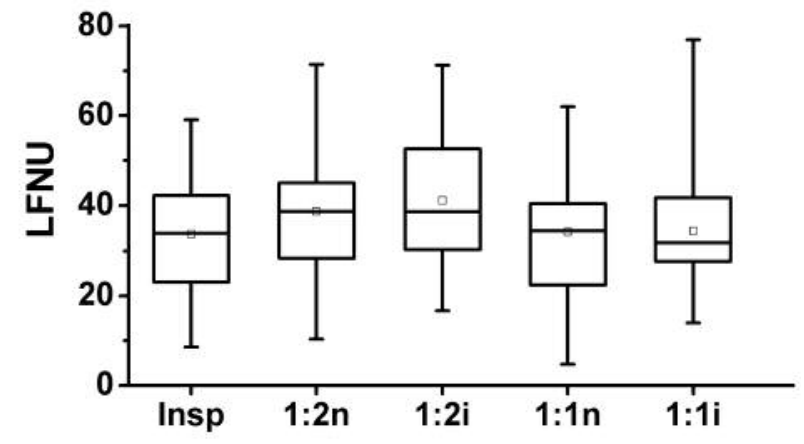

C

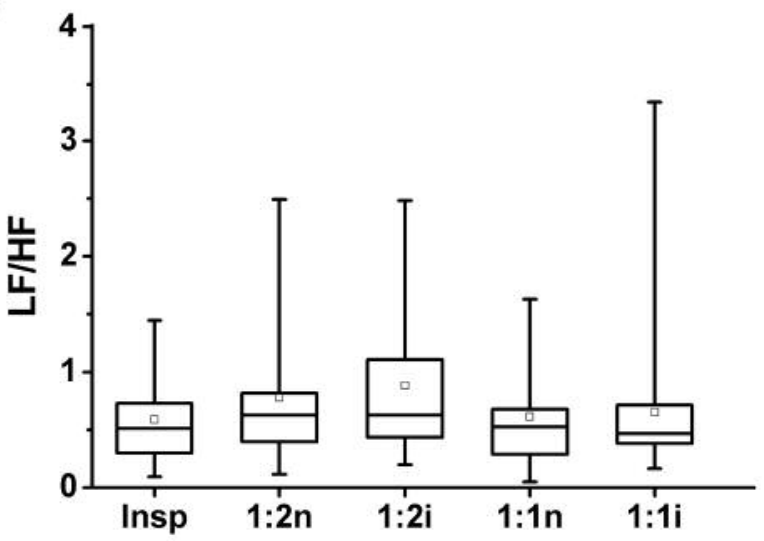

Figure 2. Box and whisker diagrams of the frequency domain parameters. A: High frequency power in normalized units (HFNU). B: Low frequency band power in normalized units (LFNU). C: Low frequency/high frequency power ratio $(L F / H F)$. Boxes: Lower and upper quartiles, horizontal line: median value, whiskers: minimum and maximum values, square: mean value. By Friedman test $p=0.107$ for each frequency domain parameter, no significant changes. Insp: Only inspiration paced without exposure; 1:1 symmetrically paced inspiration/expiration; 1:2 asymmetrically paced inspiration/expiration; $i$ : with irradiation; $n$ : without irradiation . ratio. The radiofrequency exposure from the mobile phone took place randomly either during the second and third, or during the fourth and fifth stages, blinded to both the volunteers and the person who analyzed the records. Valentini et al. stated in their metaanalysis that GSM-irradiation causes only transient cortical phenomena by EEG studies, therefore only the instantaneous effects of radiofrequency exposure were analysed (10). For this reason and in order to minimize the mental load of our investigation, only two 6-minute exposures were applied with 1:2 and 1:1 inspiration/ expiration ratios in contrast to the frequently applied 30-minute microwave exposure. The entire measurement needed about 45 minutes per volunteer.

Statistical analysis was prepared using the statistiXL v1.8 (statistiXL, Broadway Nedlands, Western Australia) plug-in in Microsoft Excel (Microsoft Corporation, Redmond, WA, USA). The figures were constructed with OriginPro v2017 (OriginLab Corporation, Northampton, MA, USA). Non-parametric repeatedmeasures ANOVA by Friedman was applied for primary assessment. Values of $p \leq 0.05$ were considered statistically significant. Wilcoxon paired-sample tests with the Holm-Bonferroni correction of significance level were applied post-hoc. In pairs, the following were compared: A: only inspiration controlled by metronome (Insp) with sham-exposed (i.e. not exposed) asymmetrically doubletriggered breathing $(1: 2 \mathrm{n})$; B: $1: 2 \mathrm{n}$ pattern with the pattern of nonirradiated symmetrical $(1: 1 \mathrm{n})$; C: irradiated asymmetrical (1:2i) with irradiated symmetrical breathing (1:1i); D: 1:2n with 1:2i; and E: 1:1n with $1: 1$ i.

\section{Results}

On manual checking of the ECG and respiration recordings, no records were rejected due to poor signal quality, inappropriate breathing, arrhythmia or any other reason. Moreover, spectral analysis by fast Fourier transformation clearly showed the respiratory peak at around $0.22 \mathrm{~Hz}$ for each individual.

Time domain analysis. The mean RR-interval changed significantly according to Friedman's test. Wilcoxon's paired-sample test post-hoc showed a significant difference $(p=0.002)$ between the Insp and 1:2 paced sham-exposed measurements even after Holm-Bonferroni correction. Further comparisons of the mean RR-interval showed no significant changes (Figure 1A).

The Friedman test of SDNN parameters was statistically significant (Figure 1B), post-hoc paired-sample assessment resulted in significant differences even after p-value correction between 1:2 and 1:1 breathing measurements under EMF exposure $(p=0.009)$. The other comparisons regarding SDNN demonstrated no significant changes. Interestingly, the mean and median of SDNN were separated at 1:1 breathing with EMF irradiation. The CV\% behaved similarly giving a statistically significant $p=0.008$ value on comparing $1: 2 \mathrm{i}$ with $1: 1 \mathrm{i}$ series by post-hoc Wilcoxon's paired-sample test (Figure 1C).

The RMSSD resulted in significant differences by Friedman test $(p=0.022)$ and again, only 1:2i with 1:1i series 

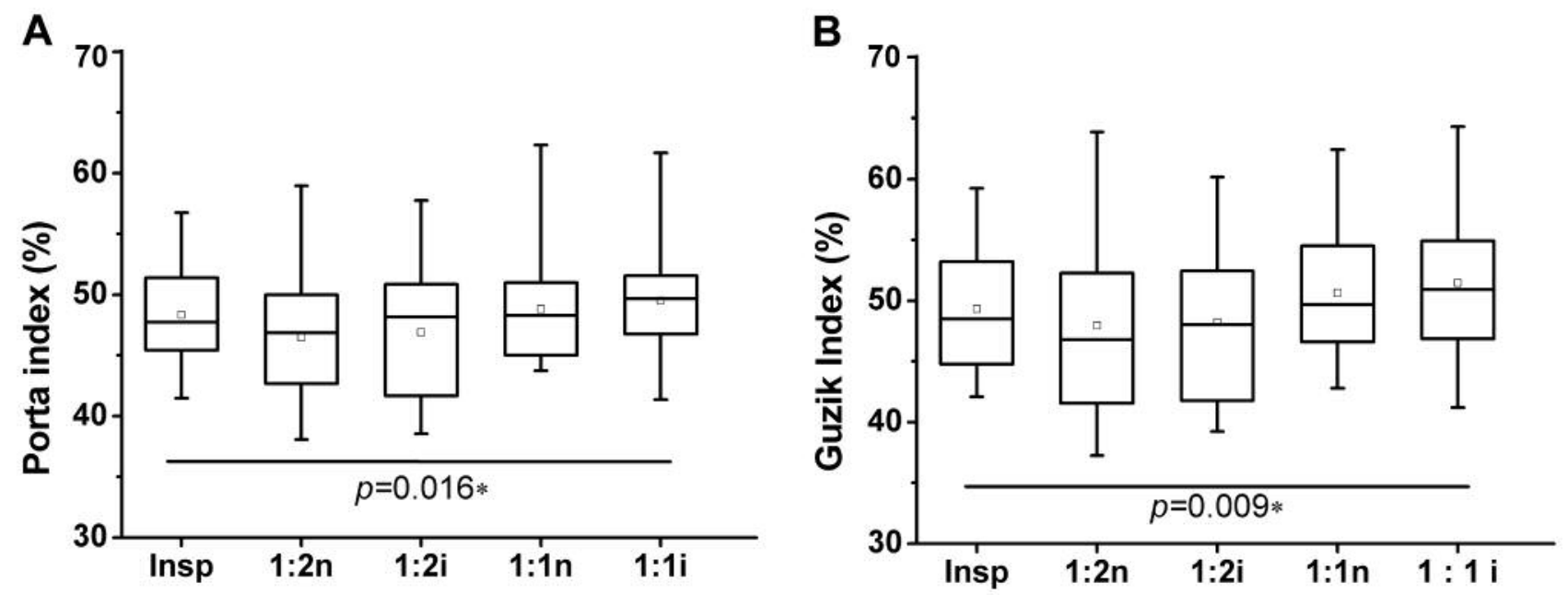

Figure 3. Box and whisker diagrams of heart rate asymmetry (HRA) parameters. A: Porta index. B: Guzik index. Boxes: Lower and upper quartiles, horizontal line: median value, whiskers: minimum and maximum values, square: mean value. *Friedman test p-value, only significant differences are indicated; the examined post-hoc Wilcoxon's paired sample tests resulted in non-significant p-values after Holm-Bonferroni correction. Insp: Only inspiration paced without exposure; 1:1 symmetrically paced inspiration/expiration; 1:2 asymmetrically paced inspiration/expiration; $i:$ with irradiation; $n$ : without irradiation.

gave significant differences by post-hoc Wilcoxon's pairedsample test $(p=0.008$, Figure 1D). The increase of the interquartile distance is evident here as well, representing a possible separation of the population during cellular phone EMF exposure at symmetrical but not asymmetrical breathing together.

Frequency domain analysis. Among frequency domain parameters (LF, HF, LF/HF) no statistically significant differences were found by Friedman test (Figure 2). Due to the complementary nature of LF and HF power and the ranking in Friedman test, the $p$-values proved to be identical.

HRA analysis. The Friedman test resulted in statistically significant $p$-values for both Porta $(p=0.016$; Figure $3 \mathrm{~A})$ and Guzik ( $p=0.009$; Figure 3B) indices. However, post-hoc Wilcoxon's paired-sample test of Porta index demonstrated only a tendency for differences between $1: 2$ and $1: 1$ breathing patterns in both comparisons sham-exposed and irradiated pairs $(p=0.011$ for $1: 2 \mathrm{n} v s .1: 1 \mathrm{n} ; p=0.024$ at $1: 2 \mathrm{i}$ for 1:1i) after Holm-Bonferroni correction of the significance level. Wilcoxon's paired-sample test for Guzik's index showed similar results: $p=0.027$ for $1: 1 \mathrm{n} v s .1: 2 \mathrm{n}$; $p=0.011$ for $1: 1 \mathrm{i} v s$. $1: 2 \mathrm{i}$. There were no significant differences in either index between sham-exposed and irradiated groups comparing the same breathing patterns: 1:1n vs. 1:1i and 1:2n vs. 1:2i (Figure 3). Both Pearson's correlation and Spearman's rank correlation confirmed a

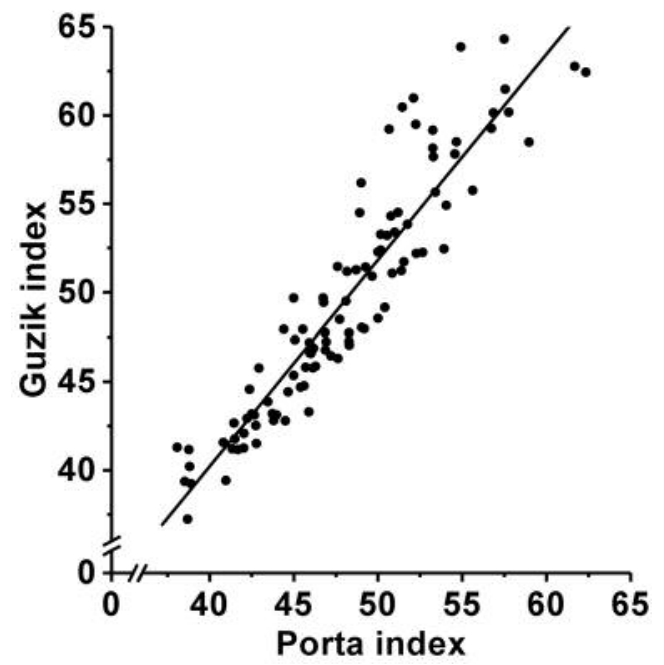

Figure 4. Pearson's correlation and Spearman's rank correlation tests between Porta and Guzik indices. The tests showed a significantly strong positive correlation. Pearson correlation: $r_{P}=0.932, p<0.0001$; Spearman rank correlation: $r_{S}=0.948, p=1.022 \times 10^{-18}$.

significantly strong positive correlation above 0.9 between Porta and Guzik indices with $p<0.001$ and $p=1.022 \times 10^{-18}$, respectively (Figure 4).

Recombination of the time domain measurements. In order to reject or confirm order-dependent effects, the five stages 


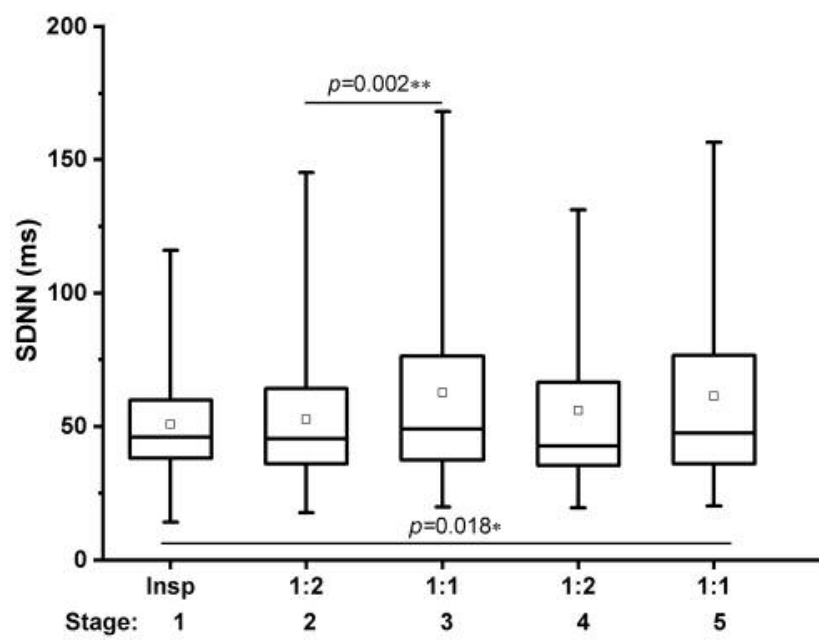

Figure 5. Standard deviation (SDNN) changes according to the order of measurement and breathing pattern. Boxes: Lower and upper quartiles, horizontal line: median value, whiskers: minimum and maximum values, square: mean value. *Friedman test p-value, **post-hoc Wilcoxon's paired-sample test p-values, only significant differences are indicated. Insp: Only inspiration paced without exposure; 1:1 symmetrically paced inspiration/expiration; 1:2 asymmetrically paced inspiration/expiration; $i$ : with irradiation; $n$ : without irradiation.

were regrouped in the order of data acquisition ignoring irradiation: 11 volunteers were exposed in the 2 nd and 3 rd stage, while nine were exposed in the 4 th and 5 th stage. Figure 5 demonstrates that there were no significant differences in SDNN among the groups with identical breathing patterns independently of the EMF exposure. Between the 1:1 and 1:2 groups, SDNN in the 2nd and 3rd stages differed, $p=0.002$ is considered a statistically significant difference even after Holm-Bonferroni correction.

Exploring possible post-exposure effects, the 2nd and 3rd irradiation $(n=11)$ and 4 th and 5th stage irradiation $(n=9)$ groups were analyzed separately according to exposure versus sham-exposure, given that RMSSD reflects parasympathetic influence on heart rate. The 2nd and 3rd stage exposed groups gave the chance to examine postexposure effects up to 12 minutes (during the 4th and 5th stages) following a 12-minute EMF exposure. During exposure $(n=11+9)$ and before exposure $(n=9)$, the $1: 1$ breathing resulted in greater median and mean RMSSD than with 1:2 breathing (Figure 6). Paradoxically, the postexposure analysis $(\mathrm{n}=11)$ showed lower mean and median RMSSD with $1: 1$ breathing. Due to the small number of people in the split groups, we did not perform further statistical analysis. However, this may be the explanation for the significant differences according to breathing patterns only between the 2 nd and 3 rd but not between the 4 th and 6th stages apparent in Figure 5.

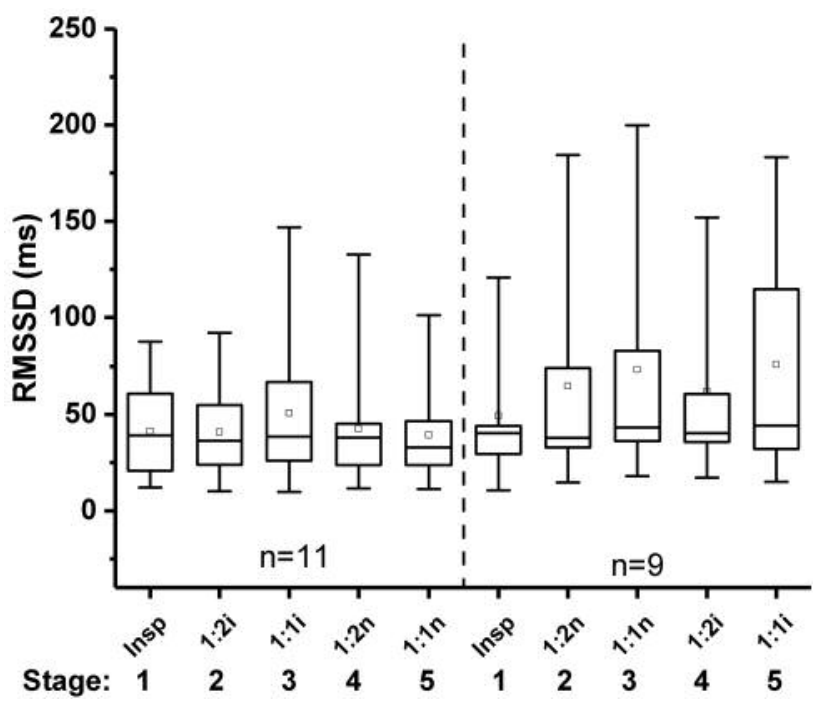

Figure 6. Root mean square of successive differences (RMSSD) changes after separation of the first-exposed and second-exposed volunteers. Boxes: Lower and upper quartiles, horizontal line: median value, whiskers: minimum and maximum values, square: mean value. Horizontal axis with the breathing patterns, the serial number, and the presence of exposure. Insp - Only inspiration paced without exposure, 1:1 symmetrically paced inspiration/expiration; 1:2 asymmetrically paced inspiration/expiration; $i$ : with irradiation; $n$ : without irradiation, bottom line: stage serial number.

\section{Discussion}

Additionally to electric inhomogeneity and anisotropy of the human body, local EMF effects are influenced by the wavelength relative to the target size, penetration/damping and intracorporal reflections of the microwave besides passive and active thermoregulation processes, such as instantaneous regional blood flow. All these factors call for sophisticated numerical simulation studies, phantom model tests, careful extrapolation of experimental results, and further epidemiological investigations $(4,6)$. In the present study, the possible effects of pulsed, low-frequencymodulated EMF emitted by mobile phones on the autonomic modulation of the heart rate was investigated by HRV and HRA analysis at different paced breathing patterns. We supposed altered breathing-respiratory sinus arrhythmia coupling on exposure which could be detected by HRA analysis. We did not find statistically significant differences in HRA parameters due to exposure, and even the earlier published (26) reporting on 1:1 vs. 1:2 breathing did not reach statistically significant differences after HolmBonferroni correction of the significance level in spite of the similar study population and pace-adhered breathing verified by recordings. The powerful correlation of Guzik and Porta indices observed in this study also calls attention to their 
redundancy, since assuming a constant heart rate during the examination period, the number of heart beats and the interbeat differences in the accelerating and decelerating set of points of the Poincaré plot are reciprocal, as detailed in our previous investigation (26).

On the other hand, we detected statistically significant elevation of the SDNN, CV\% and RMSSD parameters during irradiation in symmetrical but not in asymmetrical breathing patterns. Thus 1:1 paced breathing seems to be a sensitizing factor in HRV analysis, at least concerning the effects of EMF. The higher inter-individual variation of HRV in the 1:1i group (Figure 1B-D) may be the result of the presence of some individuals with electromagnetic hypersensitivity, or due to the non-uniform power of exposure since actual emissions during the experiment were not measured; this is a limitation of the study. The power output of mobile phones is automatically set to the necessary minimum (31) in order to increase battery life and reduce emission. Individuals with electromagnetic hypersensitivity only comprise a minority of the population: they report symptoms that may be related to exposition to weak EMF (32). However, Hietanen and co-workers did not ascribe subjective complaints of adverse effects of mobile phone emission in a blind study on 20 healthy volunteers (33). In a meta-analysis (34) no evidence was found of a causal association of electrosensitivity and EMF. Eltiti and colleagues conducted a study similar in volume on EMF-sensitive and healthy volunteers: combined GSM900, GSM1800 and UMTS $2000 \mathrm{MHz}$ base station-like exposure did not affect subjective well-being, skin conductance or pulse rate (35). Another limitation of our study is the fixed 1:2 and 1:1 sequence of breathing patterns, resulting in longer (altogether 12 minutes) exposure or a pre-exposed state at 1:1 breathing, however, we considered the negative results or only a rapidly recovering transient effect of previous authors $(10,18,23,35,36)$ regarding cellular phone exposition and HRV, hence we focused on the immediate effects of exposure on HRV and HRA parameters. However, randomization made it possible to examine post-exposure effects in 11 volunteers, where we found opposite changes of RMSSD with 1:2 versus 1:1 breathing patterns compared to the exposed (the same group $\mathrm{n}=11$ ) or pre-exposed (other group $\mathrm{n}=9$ ) cohort (Figure 6). This can reflect vagal withdrawal following 12-minute-long mobile phone EMF irradiation.

HRV analysis is a relatively frequently used non-invasive method to analyse the acute impact of mobile phone irradiation on the autonomous modulation of cardiac functions. In our previous study (23), we did not find any changes in standard HRV parameters during short-term 900 MHz GSM EMF exposure in healthy volunteers. Parazzini et al. concluded that short-term RF irradiation of healthy young persons by mobile phone does not result in statistically significant changes but suggested a weak interaction of time domain parameters and LF power (36). Other researchers came to similar conclusions regarding blood pressure and spectral HRV parameters in 120 volunteers (18). Upon habitual exposure to mobile phone radiation, the time domain HRV parameters were lower and the LF/HF ratio was higher, suggesting a shift to sympathetic activation compared to controls not using cellular phone at all (37).

The mean RR-interval at the inspiration-only controlled first run was significantly shorter (corresponding to higher pulse rate) compared to 1:2 paced sham-exposed measurements. The latter was the second stage only in about the half of the participants considering the double-blind randomized exposure. The 15 minutes of orthostatic adaptation prior to recording excludes orthostatic effects; a stress reaction caused by the beginning of the recording or starting the paced breathing might be one explanation.

Szmigielski and co-workers detected altered diurnal rhythm of blood pressure and heart rate in people regularly working exposed to EMF compared to not exposed controls with the same working pattern (16). The effect was additionally proportional to the electric field strength, ranging from 20 to $550 \mathrm{~V} / \mathrm{m}$ at $0.738-1.503 \mathrm{MHz}$. Braune and colleagues used a remote-controlled $900 \mathrm{MHz}$ GSM phone at the right hemisphere for 35 minutes in 10 healthy volunteers and found an increase in capillary vasoconstriction due to increased efferent sympathetic activity resulting in higher systolic and diastolic blood pressures but there were no changes in standard baroreflex tests (17). On the other hand, Barker et al. excluded any significant effect in mean arterial pressure and heart rate variability (HRV) parameters on short-term exposure by simulating GSM and TETRA handsets in 120 healthy subjects (18). Tahvanainen and co-workers did not find any change in arterial blood pressure or heart rate during or after 35 minutes of exposure at 900 or $1800 \mathrm{MHz}$ at maximal allowed antenna power in a double-blind placebo-controlled crossover trial in 32 healthy volunteers (19).

Braune et al. suspected blood pressure elevation and increased efferent sympathetic activity due to cellular phone irradiation central effects since there were no changes in standard baroreflex tests (17). Our experimental setup demands more complex interaction between auditory (beep for pacing), cortical (recognition and differentiation of beep frequency and intentional breathing in and out), and autonomic centres (intentional breathing and reflex responses to intrathoracal and blood pressure changes), all of them localized in the vicinity of the microwave signal source. Valentini and co-workers concluded in their metaanalysis of the neurophysiological effects of mobile phone exposure that not only the cortex but also subcortical structures such as the hypothalamus and thalamus can be affected by pulsed microwave radiation since the alpha and sigma bands of EEG are enhanced during exposure (10). However, in our study we cannot exclude the role of 
baroreflexes following intrathoracal pressure changes due to breathing either at the receptor level or at processing in the brain stem. In addition, the carotid body is close to a mobile phone during use, so its function may also vary with exposure. We believe an interaction exists between the affected centres resulting in a delay of feedback which can increase HRV under 1:1 breathing and EMF exposure. This might be due to the excitation effect of cellular phone exposure on the brain (10), resulting in increased sympathetic activity in accordance with Braune et al. (17) and Ekici and co-workers (37), considering also the relatively longer inspiratory period during the $1: 1$ breathing compared to the $1: 2$ pattern. During inhalation, respiratory sinus acceleration is a result of vagal withdrawal and increased sympathetic flow. In our investigation, HF power (vagal effect) decreased and LF power (vagal and sympathetic interactions) increased upon exposure during 1:2 breathing (Figure 2), however, it did not reach statistical significance by the Friedman test. We suspect that microwave exposure acts mainly during inspiration.

\section{Conclusion}

To our knowledge, the present examination was the first investigating HRA and HRV parameters with different paced inspiration/expiration ratios during short-term GSM cellular phone exposure in a double-blind repeatedmeasures design crossover trial. Significant HRA changes were not found on exposure compared to sham exposure, however, time domain HRV parameters on exposure were significantly different at 1:1 paced but not at 1:2 paced breathing, considering symmetrical breathing as a sensitizing factor. We observed a mild post-exposure effect regarding RMSSD. This finding reflects persisting acute effects of GSM handset emission on the autonomic nervous system, although exploring its real effects on health status and on survival, as well as the elucidation of any underlying physiopathology, call for further highervolume double-blind studies focused on respirationcirculation coupling. Our study reproduced the HRA changes accordingly to the metronome-controlled inspiration/expiration ratios. The strong correlation of Porta and Guzik indices was also demonstrated in the overall study raising their redundant nature. Symmetrical breathing (1:1 inspiration:expiration ratio) can be used as sensitizing factor or 'stress test' in other HRV/HRA analysis studies.

\section{Conflicts of Interest}

The Authors declare that they have no conflict of interest in regard to this study.

\section{Funding}

OriginPro v2017 software was funded by 3/2016 AOK-KA grant from the Medical Faculty of the University of Pécs, Hungary. Balázs Németh was supported by the ÚNKP-17-3-III New National Excellence Program of the Ministry of Human Capacities, Hungary.

\section{Ethical Approval}

All procedures performed in the study were in accordance with the ethical standards of the Regional Research Ethics Committee and with the 1964 Helsinki declaration and its later amendments.

\section{Acknowledgements}

The Authors express thanks to Levente Nyitrai, former student, for his assistance in data acquisition and analysis. The present scientific contribution is dedicated to the 650th anniversary of the foundation of the University of Pécs, Hungary.

\section{References}

1 Funk RH, Monsees T and Ozkucur N: Electromagnetic effects From cell biology to medicine. Prog Histochem Cytochem. 43(4): 177-264, 2009.

2 GSMA. About us. History - Brief history of GSM and the GSMA. Available from: https://www.gsma.com/aboutus/history Last accessed on 28 September 2017.

3 Maros D and Temesvári Z: Capacity of mobile networks in emergency case. Hadmérnök (The War Engineer) XII/1: 247254, 2017.

4 Mátay G and Zombory L: Physiological effects and biomedical applications of radiofrequency radiation. Budapest, Múegyetemi Kiadó, pp. 31-148, 2000. (Hungarian).

5 Szalay L and Ringler A: Biophysics. Budapest, Tankönyvkiadó, pp. 126-138, 1985.

6 Stavroulakis P: Mechanisms of Action of EMFs on Biological Systems. Introduction. In: Biological Effects of Electromagnetic Fields. Stavroulakis P (ed). Springer-Verlag, Berlin-Heidelberg, p. 4, 2003.

7 Kim KE, Park SK, Nam SY, Han TJ and Cho IY: Potential therapeutic mechanism of extremely low-frequency high-voltage electric fields in cells. Technol Health Care 24(3): 415-427, 2016.

8 William AS: Techmind. GSM phone signal analysis. Available from: http://www.techmind.org/gsm/ Last accessed on 28 September 2017.

9 World Health Organisation (WHO). Global Health Observatory data repository. Exposure limits for radio-frequency fields (public). Data by country. Available from: http://apps.who.int/ gho/data/view.main.EMFLIMITSPUBCRADIOFREQUENCYv Last accessed on 28 September 2017.

10 Valentini E, Curcio G, Moroni F, Ferrara M, De Gennaro L and Bertini M: Neurophysiological effects of mobile phone electromagnetic fields on humans: a comprehensive review. Bioelectromagnetics 28(6): 415-432, 2007.

11 Krause CM, Sillanmaki L, Koivisto M, Haggqvist A, Saarela C, Revonsuo A, Laine $M$ and Hamalainen $H$ : Effects of electromagnetic field emitted by cellular phones on the EEG during a memory task. Neuroreport 11(4): 761-764, 2000. 
12 Koivisto M, Revonsuo A, Krause C, Haarala C, Sillanmaki L, Laine $\mathrm{M}$ and Hamalainen $\mathrm{H}$ : Effects of $902 \mathrm{MHz}$ electromagnetic field emitted by cellular telephones on response times in humans. Neuroreport 11(2): 413-415, 2000.

13 Croft RJ, Chandler JS, Burgess AP, Barry RJ, Williams JD and Clark AR: Acute mobile phone operation affects neural function in humans. Clin Neurophysiol 113(10): 1623-1632, 2002.

14 Preece AW, Iwi G, Davies-Smith A, Wesnes K, Butler S, Lim E and Varey A: Effect of a $915-\mathrm{MHz}$ simulated mobile phone signal on cognitive function in man. Int J Radiat Biol 75(4): $447-$ 456, 1999.

15 Ferreri F, Curcio G, Pasqualetti P, Gennaro LD, Fini R and Rossini PM: Mobile phone emissions and human brain excitability. Ann Neurol 60(2): 188-196, 2006.

16 Szmigielski S, Bortkiewicz A, Gadzicka E, Zmyslony M and Kubacki R: Alteration of diurnal rhythms of blood pressure and heart rate to workers exposed to radiofrequency electromagnetic fields. Blood Press Monit 3(6): 323-330, 1998.

17 Braune S, Wrocklage C, Raczek J, Gailus T and Lucking CH: Resting blood pressure increase during exposure to a radiofrequency electromagnetic field. Lancet 351(9119): 1857-1858, 1998.

18 Barker AT, Jackson PR, Parry H, Coulton LA, Cook GG and Wood SM: The effect of GSM and TETRA mobile handset signals on blood pressure, catechol levels and heart rate variability. Bioelectromagnetics 28(6): 433-438, 2007.

19 Tahvanainen K, Nino J, Halonen P, Kuusela T, Laitinen T, Lansimies E, Hartikainen J, Hietanen $M$ and Lindholm $\mathrm{H}$ : Cellular phone use does not acutely affect blood pressure or heart rate of humans. Bioelectromagnetics 25(2): 73-83, 2004.

20 Chattipakorn N, Incharoen T, Kanlop N and Chattipakorn S: Heart rate variability in myocardial infarction and heart failure. Int J Cardiol 120(3): 289-296, 2007.

21 Hejjel L and Gal I: Heart rate variability analysis. Acta Physiol Hung 88(3-4): 219-230, 2001.

22 Task Force: Heart rate variability: Standards of measurements physiological interpretation and clinical use. Task Force of ESC/NASPE (European Society of Cardiology/North American Society of Pacing and Electrophysiology). Circulation 93: 1043$1065,1996$.

23 Atlasz T, Kellenyi L, Kovacs P, Babai N, Thuroczy G, Hejjel L and Hernadi I: The application of surface plethysmography for heart rate variability analysis after GSM radiofrequency exposure. J Biochem Biophys Methods 69(1-2): 233-236, 2006.

24 Guzik P, Piskorski J, Krauze T, Wykretowicz A and Wysocki H: Heart rate asymmetry by Poincaré plots of RR intervals. Biomed Tech 51(4): 272-275, 2006.

25 Porta A, Casali KR, Casali AG, Gnecchi-Ruscone T, Tobaldini E, Montano N, Lange S, Geue D, Cysarz D and Van Leeuwen P: Temporal asymmetries of short-term heart period variability are linked to autonomic regulation. Am J Physiol Regul Integr Comp Physiol 295(2): 550-557, 2008.

26 Klintworth A, Ajtay Z, Paljunite A, Szabados S and Hejjel L: Heart rate asymmetry follows the inspiration/expiration ratio in healthy volunteers. Physiol Meas 33(10): 1717-1731, 2012.
27 Nokia 6230i user guide. 2006; Issue 3. Available from: www.rtv.co.uk/users_manuals/Nokia_6230i_UG_en.pdf. Last accessed on 28 September 2017.

28 Bernardi L, Wdowczyk-Szulc J, Valenti C, Castoldi S, Passino C, Spadacini G and Sleight P: Effects of controlled breathing, mental activity and mental stress with or without verbalization on heart rate variability. J Am Coll Cardiol 35(6): 1462-1469, 2000.

29 Pinna GD, Maestri R, La Rovere MT, Gobbi E and Fanfulla F: Effect of paced breathing on ventilatory and cardiovascular variability parameters during short-term investigations of autonomic function. Am J Physiol Heart Circ Physiol 290(1): H424-433, 2006.

30 Stark R, Schienle A, Walter B and Vaitl D: Effects of paced respiration on heart period and heart period variability. Psychophysiology 37(3): 302-309, 2000.

31 Lönn S, Forssén U, Vecchia P, Ahlbom A and Feychting M: Output power levels from mobile phones in different geographical areas; implications for exposure assessment. Occup Environ Med 61(9): 769-772, 2004.

32 Bergqvist U, Vogel E, Aringer L, Cunningham J, Gobba F, Leitgeb N, Miro L, Neubauer G, Ruppe I, Vecchia P and Wadman C: Possible health implications of subjective symptoms and electromagnetic fields. A report prepared by a European group of experts for the European Commission. DG V. Solna: Arbete och Halsa; 1997. https://gupea.ub.gu.se/bitstream/ 2077/4156/1/ah1997_19.pdf accessed on 12 June 2017.

33 Hietanen M, Hamalainen AM and Husman T: Hypersensitivity symptoms associated with exposure to cellular telephones: no causal link. Bioelectromagnetics 23(4): 264-270, 2002.

34 Rubin GJ, Das Munshi J and Wessely S: Electromagnetic hypersensitivity: a systematic review of provocation studies. Psychosom Med 67(2): 224-232, 2005.

35 Eltiti S, Wallace D, Ridgewell A, Zougkou K, Russo R, Sepulveda F, Mirshekar-Syahkal D, Rasor P, Deeble R and Fox E: Does short-term exposure to mobile phone base station signals increase symptoms in individuals who report sensitivity to electromagnetic fields? A double-blind randomized provocation study. Environ Health Perspect 115(11): 1603-1608, 2007.

36 Parazzini M, Ravazzani P, Tognola G, Thuroczy G, Molnar FB, Sacchettini A, Ardesi G and Mainardi LT: Electromagnetic fields which are produced by GSM cellular phones and heart rate variability. Bioelectromagnetics 28(2): 1221-29, 2007.

37 Ekici B, Tanindi A, Ekici G and Diker E: The effects of the duration of mobile phone use on heart rate variability parameters in healthy subjects. Anatol J Cardiol 16(11): 833-838, 2016.

Received April 9, 2018

Revised May 27, 2018

Accepted May 29, 2018 